\title{
Has Embase replaced MEDLINE since coverage expansion?
}

Michael Thomas Lam; Christina De Longhi, MLIS; Joseph Turnbull, PhD; Helen Rose Lam; Reena Besa, MLIS

See end of article for authors' affiliations.

Objectives: The research tested the authors' hypothesis that more researchers from the academic medicine community in the United States and Canada with institutional access to Embase had started using Embase to replace MEDLINE since Embase was expanded in 2010 to cover all MEDLINE records.

\begin{abstract}
Methods: We contacted libraries of 140 and 17 medical schools in the United States and Canada, respectively, to confirm their subscriptions to Embase 5 years before and 5 years after 2010. We searched the names of institutions with confirmed Embase access in Ovid MEDLINE and Embase to retrieve works authored by affiliates of those institutions. We then examined 100 randomly selected records from each of the 5 years before and 5 years after the Embase coverage expansion in 2010. We hypothesized that studies that used Embase but not MEDLINE would increase due to the Embase coverage expansion.
\end{abstract}

Results: The number of studies that used Embase but not MEDLINE did not change between the pre-2010 and post-2010 periods.

Conclusion: Our hypothesis was refuted. Studies that used Embase but not MEDLINE did not increase post2010. Our results suggest the academic medicine community in the United States and Canada that had access did not use Embase to replace MEDLINE, despite the Embase coverage expansion.

\section{INTRODUCTION}

Many types of biomedical research require searching the medical literature as part of the research process to understand the current evidence or knowledgebase. This is true when conducting primary research such as basic science or laboratory research, clinical research, and epidemiological research as well as secondary research such as narrative reviews, systematic reviews, and metaanalyses [1]. This is usually done using one or more purpose-built biomedical bibliographic database such as MEDLINE or Embase. Despite the advent of computerized searching offered since the early 1970s by MEDLINE (MEDLARS Online) and Embase (Excerpta Medica) [2], the top two most preferred biomedical databases for decades [3], research remains challenging due to the sheer volume of the medical literature $[4,5]$ and complexity of medical topics and terminologies, as well as possible errors in the databases [6].
It is widely recognized that a search in MEDLINE (free via PubMed, paid subscription via Ovid, and other interfaces) alone does not provide comprehensive coverage of the existing literature [79]. The Cochrane Collaboration recommends that authors of systematic reviews search at least Embase and Cochrane Central in addition to MEDLINE [10]. According to a study done in 2016, MEDLINE was the most popular database among studies that searched 1 or multiple databases [3]. MEDLINE was estimated to have contained 24.3 million records in 2015 [11].

In research studies that used multiple databases, Embase was the second most popular database after MEDLINE [3]. Embase, estimated to contain 29.9 million records near the end of 2014 [12], was also widely used for research on drug-related topics, as studies have shown the database offers better coverage than MEDLINE on pharmaceutics-related literature [13-15]. Some authors also recommended 
Embase when researching certain subtopics in health care, such as complementary and alternative medicine, prognostic studies, telemedicine, psychiatry, or health technology [16-20], making it a costly but versatile supplement and competitor to MEDLINE [2, 21].

Historically, MEDLINE and Embase were reported to have a coverage overlap ranging from $34 \%$ [22] to $70 \%$ [23]. In 2011, the Cochrane Collaboration claimed in version 5.1 of their handbook that MEDLINE and Embase each had approximately 1,800 unique journal titles that were not covered by the other database [10]. In 2017, Elsevier, the parent company of Embase, suggested that Embase had 2,800 unique journal titles, while MEDLINE had 2,500, with the 2 databases sharing 3,000 common titles [24]. Despite the variations among reports regarding common coverage at different times, an equivalent search in both MEDLINE and Embase has always been recognized as necessary when comprehensive coverage is required $[8,22,25,26]$.

In 2010, Embase underwent an ambitious project to include all MEDLINE citations in Embase (Figure 1). Unique MEDLINE records are imported into Embase on an ongoing basis after mapping of Medical Subject Headings (MeSH) terms into Emtree terms. These MEDLINE records reside in Embase but remain different than regular Embase records due to the lack of original subject indexing by Embase [24]. Since this coverage expansion - at least in theory and without taking into consideration the different indexing practices of the two databases - a search in Embase alone should cover every record in both Embase and MEDLINE, making Embase a possible "one-stop" search engine for medical research. This raises the question of whether a separate search in MEDLINE is still necessary post2010, if Embase is available to researchers via an institution-wide subscription, because one of the major obstacles to an Embase search has been its high cost [13].

The authors designed this retrospective study to test our hypothesis that a greater percentage of researchers from the academic medicine community in the United States and Canada who have access to Embase through institutional subscriptions started using Embase more frequently to replace MEDLINE since the Embase coverage expansion in 2010.

\section{METHODS}

Between October 2016 and February 2017, we used email or telephone calls to contact the medical libraries of 117 allopathic (medical degree [MD]granting) and 23 osteopathic (doctor of osteopathic medicine [DO]-granting) medical schools and/or their parent institutions in the United States, as well as those of 17 English- and French-speaking (MD- or doctor of medicine and master of surgery [MDCM]granting) medical schools in Canada. There were another 19 allopathic and 15 osteopathic schools in the United States that we did not contact, as they did not yet exist in the year 2005, the year our study period began. Our purpose was to create a list of institutions that offered uninterrupted Embase access to their library users within the 5-year periods before (2005-2009) and after (2011-2015) the Embase coverage expansion in 2010.

We chose to examine studies authored by members of the academic medicine community, namely, medical schools' affiliates, because we hypothesized that these researchers, as a group, paid the most attention to the latest evidence in the literature and received relatively strong support from professional librarians or information specialists and, hence, were more likely to make informed decisions about their choice of databases.

We then performed a search in Ovid MEDLINE and Embase in the same search session for the names of the medical schools and/or parent institutions with confirmed Embase access, directing the keyword search to the "institution (IN)" fields of only the two databases (Table 1). The strategy continued with another search for keywords such as "MEDLINE," "PubMed," "Embase," "Excerpta Medica," "literature search," "database search," and "literature review" in the "abstract (AB)" fields to identify studies with a literature search component. We imposed no restrictions on the publication type and included case reports, clinical trials, cohort studies, practice guidelines, narrative reviews, systematic reviews, qualitative or quantitative studies, and prospective or retrospective studies, with the aim of obtaining an overview of all research activities that used a biomedical database. 
Figure 1 Coverage overlap of MEDLINE and Embase pre- and post-2010

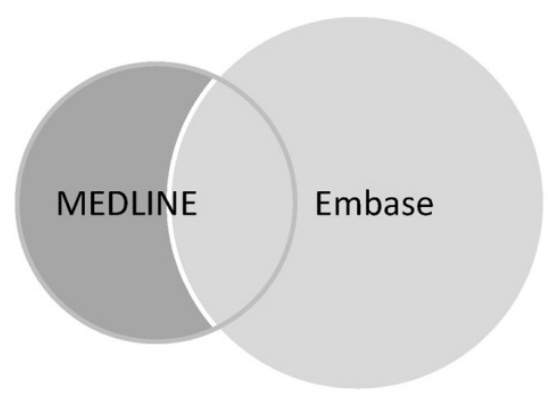

Pre-2010

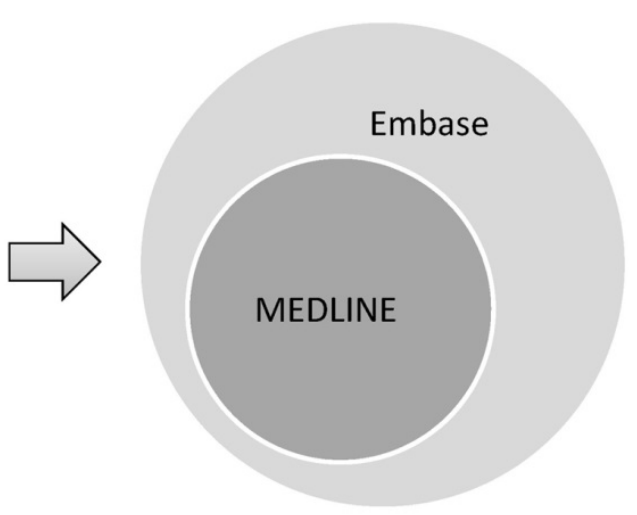

Post-2010

* Drawings not to scale.

The search was then limited to one year at a time for the five years from 2005 to 2009 (before the coverage expansion of Embase in 2010) and the five years from 2011 to 2015 (after the expansion). We excluded the year 2010 because it was a transition period, during which the uploading of MEDLINE records into Embase was still in progress. To facilitate examination of full text, we limited our search to articles with full text available from our local university library, the University of Toronto Libraries. The resulting records from the Ovid search, for each year, were rearranged into random order using randomization software [27].

We then imported the records into EndNote X8 software. Our team carefully screened the individual abstracts of these randomized records to extract information pertaining to the usage of Embase and/or MEDLINE. If the information of interest was not found in the abstracts, we examined the full text. Records that did not clearly mention using either MEDLINE or Embase in the full text were voided and excluded until 100 informative records were covered for each year. Information about the usage of databases other than MEDLINE or Embase was ignored, as the focus was on whether researchers had used Embase to replace MEDLINE (Figure 2).
Figure 2 Research process flowchart

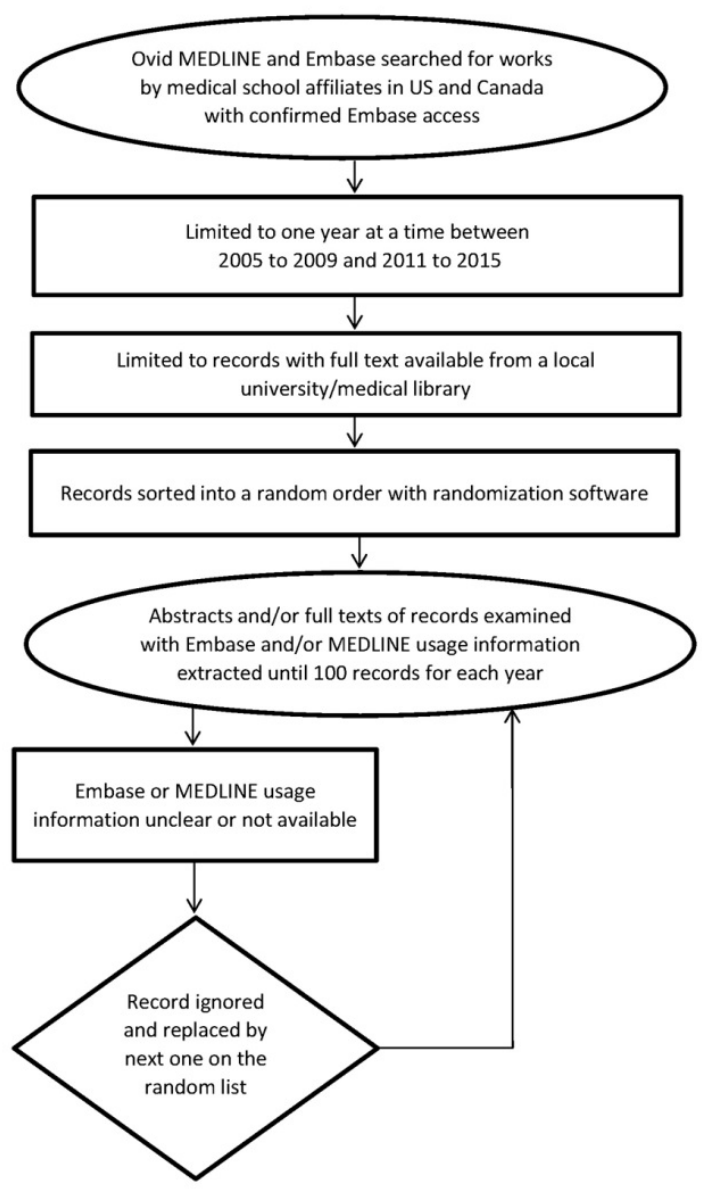


DOI: dx.doi.org/10.5195/jmla.2018.281

Table 1 Databases search strategy

\begin{tabular}{|c|c|}
\hline & Ovid MEDLINE <1946 to April Week 1 2017>, Embase Classic+Embase <1947 to 2017 April 18> \\
\hline 1 & $\begin{array}{l}\text { (McMaster University or MacMaster University or Michael G DeGroote School of Medicine or Queens } \\
\text { University or Queens School of Medicine or University of Calgary or Cumming School of Medicine or } \\
\text { UCalgary or University of Alberta or UAlberta or Western University or University of Western Ontario or } \\
\text { UWO or Schulich School of Medicine or McGill University or Universit* McGill or McGill or University of } \\
\text { British Columbia or UBC or University of Ottawa or UOttawa or Universit* d Ottawa or University of } \\
\text { Saskatchewan or USaskatchewan or Laval or Universit* Laval or Laval University or University of } \\
\text { Toronto).in. }(919,463)\end{array}$ \\
\hline 2 & 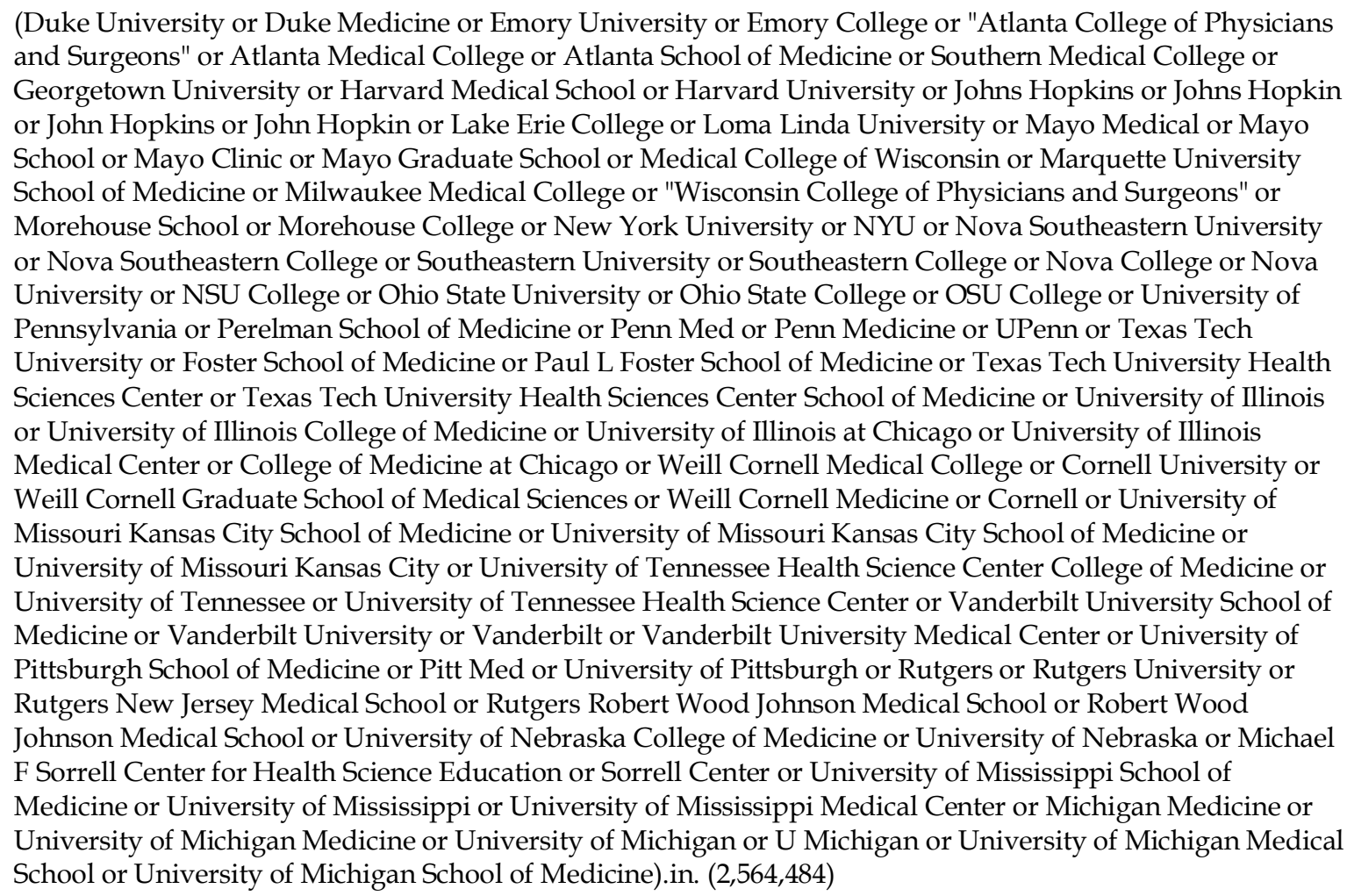 \\
\hline 3 & 1 or $2(3,439,905)$ \\
\hline 4 & $\begin{array}{l}\text { (MEDLINE or Pubmed or Embase or Excerpta Medica or ((literature or database or systematic }{ }^{*} \text { adj2 (search* } \\
\text { or review*))).ab. }(597,957)\end{array}$ \\
\hline 5 & 3 and $4(74,070)$ \\
\hline 6 & limit 5 to english language $(73,810)$ \\
\hline 7 & limit 6 to $y r=" 2015 "(10,170)$ \\
\hline 8 & remove duplicates from $7(5,606)$ \\
\hline 9 & limit 8 to full text $(1,133)$ \\
\hline
\end{tabular}

We measured three parameters for comparison between the pre- and post-2010 periods. We argue that any increase in the percentage of studies that used Embase but not MEDLINE (E) from pre-2010 (E1) to post-2010 (E2) supported our hypothesis. For reference, we also looked at the percentage of studies that used both MEDLINE and Embase (ME) and the percentage of studies that used MEDLINE but not Embase (M). While any change in these two values is of reference value, we argue that any change in these two values alone does not support or refute our hypothesis. We reason any 
abandonment of MEDLINE in favor of Embase would be clearly reflected as an increase in Embase but not MEDLINE (E).

\section{RESULTS}

We compiled a list of 39 medical schools and institutions that provided uninterrupted Embase access to their library users within the period from 2005 to 2015, representing $20.5 \%$ of US MD schools (24/117), $13.0 \%$ of US DO schools (3/23), and $70.5 \%$ of Canadian schools (12/17) that existed in 2005.

Medical schools with no or intermittent access to Embase during the period were excluded.

Upon careful examination of the 500 randomly selected records from each of the pre- and post-2010 periods, the value of $\mathrm{E}$ (Embase but not MEDLINE) decreased from $0.2 \%(\mathrm{E} 1=1 / 500)$ during the pre-2010 period to $0(\mathrm{E} 2=0 / 500)$ during the post-2010 period. For reference, the value of ME (both MEDLINE and Embase) increased from 38.8\% (ME1=194/500) to $51.4 \%$ (ME2=257/500), while the value of $\mathrm{M}$ (MEDLINE but not Embase) decreased from $61.0 \%$ (M1=305/500) to $48.6 \%$ (M2=243/500) (Table 2). We found a significant change in the distributions of the pre- and post-2010 measures of E, M, and ME over time (3 pairs of possible states compared over 2 different intervals of time, $\chi^{2}(2, n=500)=34.06$, $p<0.0001)$. Statistical sampling errors were estimated at $2 \%$ for these measures at the $95 \%$ confidence level. Thus, contrary to our initial hypothesis, we found that, within statistical error, while the value of $E$ did not increase from pre- to post-2010, a greater proportion of researchers now use both Embase and MEDLINE, and fewer rely on MEDLINE alone.

To confirm the adequacy of our sample size, we completed an analysis of another 250 independent and randomly selected records from each of the preand post-2010 periods (50 records from each year). In this smaller sample, the value of $E$ was unchanged at 0 during both time periods. Here, statistical sampling errors were estimated as 3\% for all measures at the $95 \%$ confidence level. The shifts in the ME and M proportions were also comparable to those found for the larger sample within statistical error.

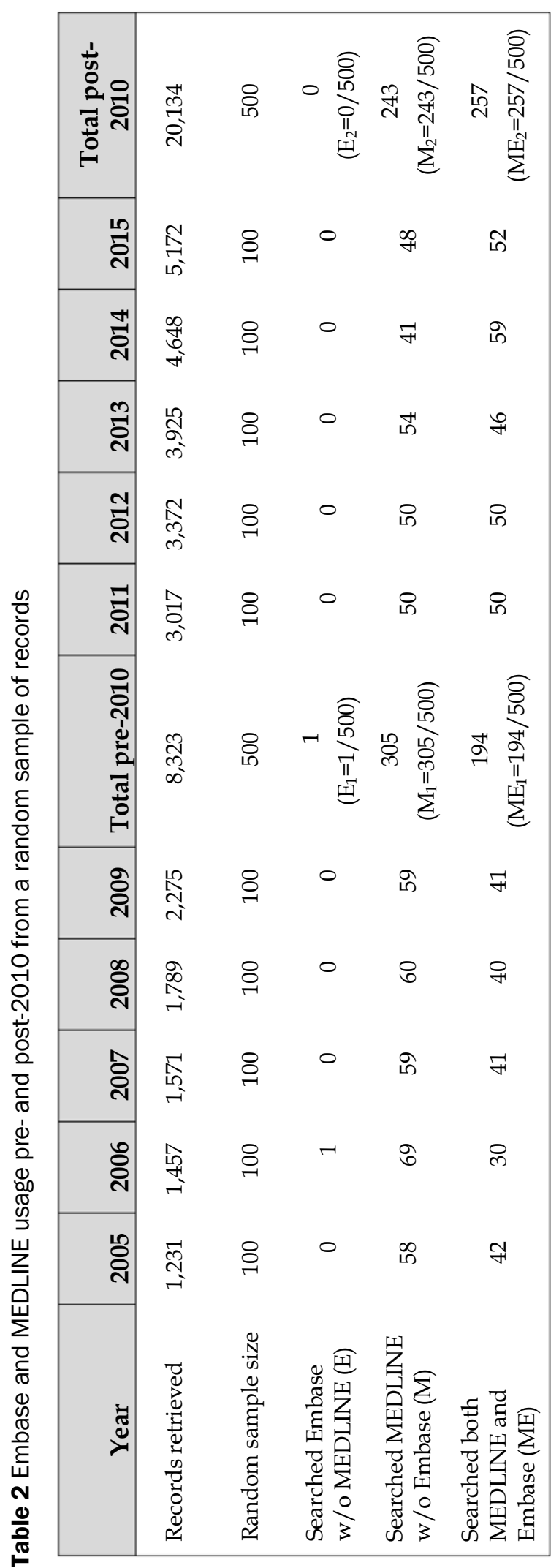




\section{DISCUSSION}

Our results suggest that, despite the Embase coverage expansion to include all of MEDLINE since 2010, researchers in the academic medicine community of the United States and Canada with access to Embase did not use Embase to replace MEDLINE.

This could be due to several reasons. While one possibility was that researchers did not know about this major coverage change in Embase, we argue that this was improbable, as researchers in this population were likely supported by professional librarians or information specialists at their institutions. Another argument against this scenario was that there has been no gradual increase in searching Embase but not MEDLINE (E) between the years 2011 and 2015. If lack of awareness about Embase coverage expansion was the reason, as information was shared and knowledge of researchers improved with time, there should have been a gradual change, which was not observed.

Another possible explanation is that researchers and their professional librarians or information specialists favored the indexing practice and quality of MEDLINE over those of Embase, either out of preference or habit. There have been reports of EMTREE terms (subject headings used in Embase) being assigned too loosely in Embase, resulting in subject searches with unnecessarily high sensitivity and low precision [28]. It is also possible that some researchers would like to cover the MEDLINE-inProcess materials and some additional publications in PubMed that are not covered promptly or completely by Embase, even after coverage expansion $[29,30]$. Another possibility is that the academic medicine community in the United States and Canada prefers a not-for-profit North American database (MEDLINE) created by a government agency over a privately owned commercial European database (Embase). The true reasons behind researchers not using Embase to its full potential are unknown and deserve further investigation.

Another trend shown in the results was a drop in M (used MEDLINE but not Embase) and an increase in ME (used both MEDLINE and Embase). Historically, most studies that searched only one database chose MEDLINE/PubMed, but this type of single database study has been in decline [3].
The results of our study suggest that researchers still view Embase and MEDLINE as separate resources to be searched individually, despite the Embase coverage expansion. Indeed, the Cochrane Collaboration did not call for a change to its recommendation for authors after the Embase expansion in 2010 [10].

We were surprised that a mere $20.5 \%$ of US MD schools and $13.0 \%$ of US DO schools provided uninterrupted access to Embase during the period from 2005 to 2015. By comparison, $70.5 \%$ of Canadian medical schools offered access during the same period. This occurred at a time when the average number of databases searched in systematic reviews was reported to be increasing, with Embase being the second-most-used resource alongside MEDLINE/PubMed [3]. The high cost of Embase and the lack of awareness of the importance of Embase among the schools' decision-makers might be factors leading to the low subscription rate among medical school libraries in the United States.

A limitation of our study is that we restricted the search results to records with full text available from the University of Toronto Libraries system. We did this to have the capacity to examine the full text of each record, when needed, without paying for interlibrary loan orders. This might have resulted in selection bias toward studies published in relatively well-established journals that were more likely to be available in the major university medical library we accessed. Hence, our study results are representative of this particular collection and might or might not be representative of all records in the databases. Despite this limitation, however, we believe our research results are of reference value to information professionals and medical researchers.

\section{REFERENCES}

1. Röhrig B, Prel JBd, Wachtlin D, Blettner M. Types of study in medical research. Dtsch Arztebl Int. 2009 Apr;106(15):262-8.

2. Blanken RR, Vinken PJ. Medical databases: Medline vs. Excerpta Medica. Inf Serv Use. 2001 Apr;21(2):87-97.

3. Lam MT, McDiarmid M. Increasing number of databases searched in systematic reviews and meta-analyses between 1994 and 2014. J Med Libr Assoc. 2016 Oct;104(4):284-9. DOI: http://dx.doi.org/10.3163/1536-5050.104.4.006.

4. US National Library of Medicine. Key MEDLINE indicators [Internet]. Washington, DC: National Institutes of Health; 2016 [cited 26 Jan 2018]. <https://www.nlm.nih.gov/bsd/bsd_key.html>. 
5. US National Library of Medicine. Fact sheet: MEDLINE [Internet]. Washington, DC: National Institutes of Health; 2016 [cited 26 Jan 2018].

<https://www.nlm.nih.gov/pubs/factsheets/medline.html>.

6. Cappell MS. Major bibliographic errors in PubMed: personal experience among 240 publications and proposed remediation process for errors. J Med Libr Assoc. 2016 Jan;104(1):83-4. DOI: http:// dx.doi.org/10.3163/15365050.104.1.015.

7. Dunikowski LG. EMBASE and MEDLINE searches. Can Fam Physician. 2005 Sep;51:1191.

8. Sampson M, Barrowman NJ, Moher D, Klassen TP, Pham B, Platt R, St John PD, Viola R, Raina P. Should meta-analysts search Embase in addition to Medline? J Clin Epidemiol. 2003 Oct;56(10):943-55.

9. Tatsioni A, Ioannidis J, Schmid C, Lau S. Contributions of EMBASE search in meta-analysis of diagnostic studies [abstract]. 12th Cochrane Colloquium: Bridging the Gaps; Ottawa, ON, Canada; 2004. p. 194-5.

10. Higgins JPT, Green S. Cochrane handbook for systematic reviews of interventions [Internet]. Version 5.2. Cochrane Collaboration; 2017 [cited 26 Jan 2018]. <http://training.cochrane.org/handbook>.

11. US National Library of Medicine. 2015 MEDLINE®/PubMed® baseline distribution [Internet]. Washington, DC: National Institutes of Health; 2015 [cited 26 Jan 2018].

<https://www.nlm.nih.gov/bsd/licensee/2015_stats/base line_doc.html $>$.

12. Elsevier. Embase indexing guide 2015: a comprehensive guide to Embase indexing policy [Internet]. Amsterdam, The Netherlands: Elsevier; 2015 [cited 26 Jan 2018]. <https://www.elsevier.com/_data/assets/pdf_file/0016 /92104/Embase-indexing-guide-2015.pdf>.

13. Brown CM. The benefits of searching EMBASE versus MEDLINE for pharmaceutical information. Online CDROM Rev. 1998;22(1):3-8.

14. Mogig ZD. EMBASE, the Excerpta Medica databases: quick and comprehensive drug information. Bibliotheca Medica Canadiana. 1995;16(4):152-4.

15. Houghton B, Webster VAD, Smith J. A comparison of Excerpta Medica and Medline for the provision of drug information to health care professionals. Sixth International Online Information Meeting London; 7-9 Dec 1982. Oxford, UK: Learned Information; 1982 p. 115-7s.

16. Boehm K, Raak C, Vollmar HC, Ostermann T. An overview of 45 published database resources for complementary and alternative medicine. Health Inf Libr J. 2010 Jun;27(2):93105.

17. Bahaadinbeigy K, Yogesan $\mathrm{K}$, Wootton R. MEDLINE versus EMBASE and CINAHL for telemedicine searches. Telemed J E Health. 2010 Oct;16(8):916-9.

18. McDonald S, Taylor L, Adams C. Searching the right database. a comparison of four databases for psychiatry journals. Health Libr Rev. 1999 Sep;16(3):151-6.
19. Wilczynski NL, Haynes RB. Optimal search strategies for detecting clinically sound prognostic studies in EMBASE: an analytic survey. J Am Med Inform Assoc. 2005 JulAug;12(4):481-5.

20. Lorenzetti DL, Topfer LA, Dennett L, Clement F. Value of databases other than MEDLINE for rapid health technology assessments. Int J Technol Assess Health Care. 2014 Apr;30(2):173-8.

21. Highbeam Research. EMBASE: a healthy MEDLINE alternative? Searcher. 1996;4:23.

22. Smith BJ, Darzins PJ, Quinn M, Heller RF. Modern methods of searching the medical literature. Med J Aust. $1992 \mathrm{Nov}$ 2;157(9):603-11.

23. Yonker VA, Young KP, Beecham SK, Horwitz S, Cousin K. Coverage and overlaps in bibliographic databases relevant to forensic medicine: a comparative analysis of MEDLINE. Bull Med Libr Assoc. 1990 Jan;78(1):49-56.

24. Elsevier Science. FAQs: how does Embase cover MEDLINE content [Internet]. Amsterdam, The Netherlands: Elsevier; 2017 [cited 26 Jan 2018].

$<$ https://www.elsevier.com/solutions/embasebiomedical-research/learn-and-support>.

25. Higgins JPT, Green S. Cochrane handbook for systematic reviews of interventions: MEDLINE and EMBASE [Internet]. Version 5.1.0: part 2: 6.2.1.3. Cochrane Collaboration; 2011 [10 Apr 2017; cited 26 Jan 2018]. <http://handbook-5-1.cochrane.org/>.

26. Belbenoit-Avich P. Comparing the main biomedical bibliographic databases. Inf Manag Rep. 1992;Jul:1-5.

27. Dallal GE. Welcome to Randomization.com!!! [Internet]. Randomization.com; 2013 [cited 26 Jan 2018]. <http://randomization.com>.

28. Glanville J, Kaunelis D, Mensinkai S, Picheca L. Pruning Emtree: does focusing Embase subject headings impact search strategy precision and sensitivity? Ottawa, ON, Canada: Canadian Agency for Drugs and Technologies in Health; 2015.

29. Duffy S, de Kock S, Misso K, Noake C, Ross J, Stirk L. Supplementary searches of PubMed to improve currency of MEDLINE and MEDLINE In-Process searches via Ovid. J Med Libr Assoc. 2016 Oct;104(4):309-12. DOI: http://dx.doi.org/10.3163/1536-5050.104.4.011.

30. US National Library of Medicine. Fact sheet: MEDLINE, PubMed, and PMC (PubMed Central): how are they different? [Internet]. MD: Bethesda: National Institutes of Health; 2017 [cited 26 Jan 2018]. <https://www.nlm.nih.gov/pubs/factsheets/dif_med_pu b.html >. 
DOI: dx.doi.org/10.5195/jmla.2018.281

\section{AUTHORS' AFFILIATIONS}

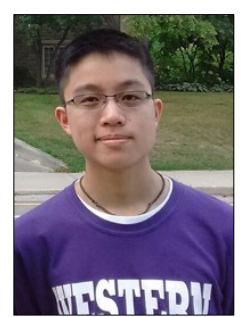

Michael Thomas Lam,

michaelthomaslam@gmail.com, Undergraduate Student, Bachelor of Medical Science Program,

Western University, London, ON, Canada

\section{Christina De Longhi, MLIS,}

mary.christina.delonghi@gmail.com,

Information Specialist, Library Services,

Sunnybrook Health Sciences Centre, Toronto,

ON, Canada

Joseph Turnbull, PhD, jturnbu7@uwo.ca, Assistant Professor of

Mathematics, Department of Economics, Business, and

Mathematics, King's University College at Western University,

London, ON, Canada

Helen Rose Lam, helenroselam@gmail.com, Undergraduate Student, Bachelor of Social Work Program, Faculty of Liberal Arts and

Professional Studies, York University, Toronto, ON, Canada

Reena Besa, MLIS, reena.besa@gmail.com, Information Specialist, Library Services, Sunnybrook Health Sciences Centre, Toronto, ON, Canada 